\title{
Comparison of Smal-defined genotypes of Campylobacter jejuni examined by Kpnl: a population-based study
}

\author{
SOPHIE MICHAUD*\&, SUZANNE MENARD $\dagger$, CHRISTIANE GAUDREAU: and \\ ROBERT D. ARBEIT§
}

* Centre Hospitalier Universitaire de Sherbrooke and †Direction de la Santé Publique de l'Estrie, Sherbrooke,
†entre Hospitalier de I'Université de Montreal, Hôpital St-Luc, Montréal, Québec, Canada; §Research Service,
VA Medical Center, and Department of Medicine, Boston University School of Medicine, Boston, MA, USA

\begin{abstract}
Pulsed-field gel electrophoresis (PFGE) was used to analyse 147 isolates collected in two regions of Québec province (Estrie and Montréal) between March 1998 and Feb. 1999, to determine the utility of molecular strain typing for a population-based collection of Campylobacter jejuni and to compare directly the discriminatory power of SmaI and Kpn I restriction digests. With a combination of epidemiological criteria including space and time plus molecular strain typing, $49 \%$ of isolates from Estrie and $39 \%$ of isolates from Montréal were identified as belonging to a putative cluster. For $41 \%$ of the cases, sources were either missing or explicitly unknown; the remaining sources were subject to recall bias. Thus, the evaluation of sporadic cases of campylobacter enteritis by descriptive clinical investigation alone is neither sensitive nor reliable for identifying sources of infection. In the PFGE analysis, KpnI digests provided appreciably greater discriminatory power than Sma I digests. When combining the PFGE analyses with basic epidemiological criteria, $30 \%$ of the putative $S m a$ I clusters were inconsistent with the epidemiological criteria compared with $17 \%$ of the Kpn I clusters. Among the 98 isolates assigned to clusters by $S m a$ I, only $65 \%$ gave concordant results with $K p n$ I. In contrast, among the 81 isolates assigned to clusters by $K p n I, 92 \%$ gave concordant results with Sma I. Finally, clusters that were epidemiologically related to ingestion of raw milk and specific water sources correlated better with the typing results based on KpnI than $S m a$ I. Thus, Kpn I is the enzyme of choice for molecular epidemiology studies of $C$. jejuni. The combination of continuous epidemiological surveillance and molecular strain typing may be useful for identifying new sources and mechanisms of transmission for community-acquired $C$. jejuni infection and ultimately for developing new approaches to prevention.
\end{abstract}

\section{Introduction}

Campylobacter enteritis is the third most common notifiable infectious disease in Québec province. In 1998, the number of diarrhoeal illnesses in Québec attributed to campylobacter enteritis exceeded the combined total caused by Salmonella and Shigella species, Escherichia coli O157:H7 and Yersinia enterocolitica. Between 1990 and 1998, the annual reported incidence of campylobacter enteritis increased from 29.9 to 43.4 per 100000 population. The reasons

Received 20 March 2000; revised manuscript received 22 Feb. 2001; accepted 16 May 2001.

Corresponding author: Dr S. Michaud (e-mail: smichaud@, courrier.usherb.ca). for this increase are not well defined; moreover, surveillance strategies vary among different regions within Québec province. In Estrie, all cases reported to the public health service are prospectively investigated by clinical nurses by means of a standardised questionnaire. Other regions, such as Montréal, do not routinely investigate reported cases.

Campylobacter jejuni is a commensal of the intestinal tract of a wide range of birds and mammals, including pets and domestic animals widely used for food production [1]. It is principally a food-borne pathogen, with well documented outbreaks related to ingestion of raw milk, untreated water and undercooked poultry [2]. However, most cases of campylobacter enteritis are deemed to be sporadic $[3,4]$. 
The wide distribution of campylobacters makes it difficult to identify specific sources of enteritis by traditional epidemiological investigation. Molecular strain typing methods may help clarify the complex epidemiology of these infections. Pulsed-field gel electrophoresis (PFGE) of DNA digests has proved to be a highly reproducible and discriminatory typing technique for a range of bacterial pathogens [5]. Although there are several reports describing PFGE typing of $C$. jejuni [6-8], no clear consensus has emerged as to whether $S m a \mathrm{I}$ or KpnI is the most effective restriction endonuclease for such studies [9]. SmaI has been used most commonly, but some investigators suggest that $K p n \mathrm{I}$ digests provide greater discriminatory power $[6,10,11]$.

This report describes the application of PFGE molecular strain typing to a large collection of $C$. jejuni enteritis isolates from Québec. The goals of the study were to determine whether such typing would facilitate the identification of common source clusters of community-acquired infection, and to compare directly the discriminatory power of $S m a \mathrm{I}$ and $K p n \mathrm{I}$ restriction digests.

\section{Materials and methods}

\section{Population}

All isolates of $C$. jejuni subsp. jejuni (hereafter $C$. jejuni) cultured at the Centre Universitaire de Santé de l'Estrie and at the Centre Hospitalier de l'Université de Montréal, Hôpital St-Luc, were collected prospectively between 1 March 1998 and 28 Feb. 1999. Clinical information about individual cases of campylobacter infection in Estrie was obtained prospectively by the Direction de la Santé Publique (DSP) de l'Estrie. For patients from Montréal only information about age, sex, date of culture and history of foreign travel was available.

\section{PFGE}

C. jejuni isolates were grown on sheep blood 5\% agar for $48 \mathrm{~h}$ at $37^{\circ} \mathrm{C}$ in a micro-aerobic atmosphere. Bacterial colonies were harvested, resuspended in $900 \mu \mathrm{l}$ of cold saline and treated with $100 \mu$ l of formaldehyde on ice for $1 \mathrm{~h}$ to inactivate endogenous nucleases [12]; bacterial suspensions were then washed three times in $1000 \mu \mathrm{l}$ of cold saline by centrifugation at $12000 \mathrm{rpm}$ and resuspended in $1000 \mu \mathrm{l}$ of saline. The optical densities of the bacterial suspensions were then adjusted to 1.9 at $405 \mathrm{~nm}$ and $625-\mu 1$ samples were gently mixed with $375 \mu \mathrm{l}$ of InCert agarose $1.5 \%$ (FMC BioProducts, Rockland, ME, USA). The resulting mixture was poured into moulds and DNA was prepared as described previously [13]. DNA in agarose plugs was digested overnight at $25^{\circ} \mathrm{C}$ with $S m a \mathrm{I}$ or at $37^{\circ} \mathrm{C}$ with $K p n \mathrm{I}$ and the resulting macrorestriction digests were electrophoresed in an agarose $1 \%$ gel in
$0.5 \times$ TBE buffer $(45 \mathrm{mM}$ Tris, $45 \mathrm{mM}$ boric acid, $1 \mathrm{mM}$ EDTA) at $200 \mathrm{~V}$ and $14^{\circ} \mathrm{C}$ with a contourclamped homogeneous electric field aparatus (CHEF Mapper or CHEF DRII System, BioRad, Hercules, CA, USA). Pulsing was ramped from 1 to $40 \mathrm{~s}$ over $22 \mathrm{~h}$ for Sma I digests and from 4 to $20 \mathrm{~s}$ over $23 \mathrm{~h}$ for Kpn I digests. The gels were stained with ethidium bromide and photographed under ultraviolet light with a digital camera.

Each gel comprised 15 lanes and included Sma I digests of Staphylococcus aureus NCTC 8325 in lanes 2, 8 and 14 as a reference standard and a Kpn I digest of $C$. jejuni strain 153B-80 in lane 13 as a reproducibility control. Lanes 1 and 15 were left blank; the remaining lanes were used for study isolates.

\section{GelCompar II analysis}

The PFGE fingerprinting patterns were analysed with GelCompar II (version 1.01 for Windows; Applied Maths, Kortrijk, Belgium). Restriction fragments were identified visually and the PFGE patterns were normalised by interpolation to the nearest reference lane. The molecular sizes of the fragments detected for the study isolates were calculated based on the fragments of $S$. aureus NCTC 8325. Only fragments in the size range 80-674 kb were analysed; smaller fragments were not consistently resolved. Optimisation and a position tolerance of $1.0 \%$ were applied. Dice similarity coefficients were calculated based on pairwise comparison of the PFGE profiles of study isolates. The matrix of coefficients was used to generate dendrograms based on the unweighted pair group method using arithmetic averages (UPGMA).

\section{Criteria for defining clusters}

Three different sets of criteria were used to define clusters of related study isolates. (i) Isolates were considered to have closely related genotypes based on molecular typing if their PFGE profiles were related at $\geqslant 0.90$, as determined by the GelCompar II analysis. (ii) Genotypically related isolates were considered to be clustered in space if they were cultured from patients whose infection was acquired in Québec province; infections acquired in a foreign country were excluded. (iii) Isolates that were genotypically and geographically related were considered to also be clustered in time if there were $<3$ months between sequential isolates.

\section{Results}

\section{Clinical epidemiology}

Among 111 cases (72 men and 39 women) reported to the DSP of Estrie, complete clinical information was available for $94(85 \%)$ patients. Clinical information was available for only a few patients from Montréal and therefore is not described here. 
The mean age of the cases was 25 years (range 10 months -85 years), with the highest proportion in the age groups $20-24$ years and $45-64$ years $(17 \%$ in each category). Cases occurred year round, with a peak incidence between July and October. The majority of patients presented with diarrhoea, abdominal pain and fever $(92 \%, 86 \%$ and $60 \%$ respectively); $25 \%$ of patients had bloody stools. Although most patients $(73 \%)$ had symptoms for $<2$ weeks, $6 \%$ reported symptoms for $\geqslant 6$ weeks. Four percent of patients were asymptomatic; all were children screened in the context of an international adoption. Overall, $47 \%$ of patients were treated with antibiotics and $12 \%$ were hospitalised for $>24 \mathrm{~h}$.

The most frequently identified suspected sources of infection were chicken (13\%), raw milk (7\%), a foreign trip $(9 \%)$, a meal outside the home $(10 \%)$, beef $(5 \%)$, contaminated water $(5 \%)$ and miscellaneous $(10 \%)$. However, the source was either missing or unknown for $41 \%$ of patients and the validity of even the suspected sources is questionable because they were not confirmed by either microbiological analysis or by a casecontrol study.

\section{Characterisation of PFGE analyses}

Of the 154 isolates available, 153 were from human stools and one from a blood culture. More than $95 \%$ of the isolates were typable by PFGE; seven isolates were excluded from the analysis because PFGE patterns could not be obtained after multiple attempts with Sma I, Kpn I or with both enzymes (two, three and two isolates, respectively). Thus, 147 isolates (88 from Estrie and 59 from Montréal) were analysed by both SmaI and KpnI. Sma I macrorestriction digests comprised 4-9 fragments in the size range analysed (674$80 \mathrm{~kb}$ ), compared with 5-12 fragments with Kpn I.
The $S$. aureus NCTC 8325 replicates were related at the level of 0.95 across 20 gels $(n=59$ lanes $)$ for Sma I digests and 0.94 among 26 gels $(n=75$ lanes $)$ for $K p n \mathrm{I}$ digests. Replicate profiles of $C$. jejuni strain 153B-80 were related at 0.94 for Sma I digests $(\mathrm{n}=16)$ and 0.96 for $K p n I$ digests $(\mathrm{n}=23)$. Thus, technical reproducibility was excellent. As suitable sets of unequivocally epidemiologically related isolates were not available, biological reproducibility could not be evaluated [5].

Among the 147 isolates resolved by Sma I, the overall genetic diversity was 0.37 , including $49(33 \%)$ isolates with unique genotypes plus 27 clusters; in contrast, $K p n$ I gave an overall diversity of 0.33 including 66 $(45 \%)$ isolates with unique genotypes plus 29 clusters.

\section{Molecular epidemiology}

Table 1 summarises the effect of different epidemiological criteria on the distribution of the size of clusters of C. jejuni isolates identified by PFGE analysis of Sma I and Kpn I digests. Molecular typing with Sma I alone defined 27 clusters comprising 98 isolates, compared with 29 clusters representing 81 isolates with Kpn I. The addition of space criteria excluded nine isolates from the clusters defined by SmaI typing, compared with two isolates among those clustered by Kpn I. The application of both space and time criteria excluded $27(28 \%)$ of the isolates and $8(30 \%)$ of the clusters putatively identified by Sma I typing, but only $14(17 \%)$ isolates and $5(17 \%)$ of the clusters defined by Kpn I typing.

The study also examined the concordance between the typing results obtained with each of the endonucleases (Table 2). As previously, isolates were designated 'genotypically related' if the similarity coefficient

Table 1. Number and size of clusters of $C$. jejuni isolates identified by PFGE of Sma I and KpnI digests with different epidemiological criteria

\begin{tabular}{lrcr}
\hline & \multicolumn{3}{c}{ Number of clusters identified by } \\
\cline { 2 - 4 } $\begin{array}{l}\text { Number of related } \\
\text { isolates in cluster }\end{array}$ & $\begin{array}{c}\text { Typing plus } \\
\text { space }\end{array}$ & $\begin{array}{c}\text { Typing plus } \\
\text { space and time }\end{array}$ \\
\hline Number of clusters with Sma I & 15 & 11 & 9 \\
2 & 2 & 3 & 4 \\
3 & 4 & 3 & 1 \\
4 & 4 & 4 & 3 \\
5 & 1 & 1 & 1 \\
8 & 1 & 1 & 1 \\
18 & 27 & 23 & 19 \\
Total & & & 15 \\
Number of clusters with Kpn I & 17 & 16 & 5 \\
2 & 7 & 7 & 1 \\
3 & 2 & 2 & 1 \\
4 & 1 & 1 & 1 \\
5 & 1 & 1 & 1 \\
6 & 1 & 1 & 24 \\
Total & 29 & 28 & \\
\hline
\end{tabular}


Table 2. Disagreement between clustering of isolates based on PFGE profiles of Kpn I and SmaI digests

\begin{tabular}{|c|c|c|c|c|}
\hline $\begin{array}{l}\text { Endonuclease } \\
\text { digest used to } \\
\text { define clusters }\end{array}$ & $\begin{array}{l}\text { Number of } \\
\text { isolates assigned } \\
\text { to clusters }\end{array}$ & $\begin{array}{l}\text { Enzyme digest } \\
\text { used to analyse } \\
\text { isolates within } \\
\text { clusters }\end{array}$ & $\begin{array}{l}\text { Number }(\%) \text { of } \\
\text { isolates with } \\
\text { concordant }^{\dagger} \text { results } \\
\text { for both enzymes }\end{array}$ & $\begin{array}{c}\text { Number }(\%) \text { of } \\
\text { isolates with } \\
\text { discordant }^{\S} \text { results } \\
\text { for the two enzymes }\end{array}$ \\
\hline Sma I & 98 & Kpn I & $64(65 \%)$ & $34(35 \%)$ \\
\hline Kpn I & 81 & Sma I & $75(92 \%)$ & $6(8 \%)$ \\
\hline
\end{tabular}

$\mathrm{p}<0.0001$, Fisher's exact test.

$*$ Clusters were defined as isolates whose restriction digests had an $\mathrm{SC} \geqslant 90 \%$.

† Concordant isolates were isolates that were assigned to the same cluster by the first enzyme and also had an $\mathrm{SC} \geqslant 80 \%$ with the second enzyme.

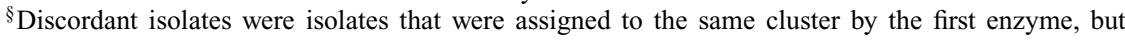
whose restriction digests had an SC $<80 \%$ with the second enzyme and were thereby assigned either to a different cluster or to no cluster.

(SC) of their PFGE profiles was $\geqslant 90 \%$. Typing results for the two enzymes were considered concordant if isolates whose PFGE profiles had a similarity coefficient of 0.90 with one endonuclease (i.e., were considered clustered) had a similarity coefficient of 0.80 when analysed with the other enzyme. Among the 98 isolates assigned to clusters by SmaI, only 64 (65\%) gave concordant results with KpnI. Among the 34 isolates with discordant results, half were assigned to other clusters and half represented unique KpnI genotypes within the study population. In contrast, among the 81 isolates assigned to clusters by Kpn I, 75 (92\%) gave concordant results with SmaI (p <0.0001, Fisher's two-tailed exact test).

Fig. 1 illustrates an example of isolates with discordant typing results. Analysis of SmaI digests of the 147 study isolates defined a subset of 49 isolates that were related at the 0.73 level (Fig. 1a) and included a single large cluster of 18 isolates related at the 0.90 level. Kpn I patterns of the same 49 isolates demonstrated considerably more diversity, with overall relatedness of 0.43 (Fig. 1b). Of note, the 18 isolates that were closely related by SmaI digests were distributed across the entire KpnI dendrogram, with six of the isolates no longer identified as closely related to any other isolate within this subgroup.

Discriminatory power was further assessed with 16 study isolates that were acquired outside Québec and, therefore, considered to be epidemiologically unrelated. The SmaI profiles of six of these isolates were closely related to isolates from Québec, compared with only one with KpnI. The overall matrix of SC indicated that pairwise comparison of each of these 16 isolates with each of the isolates from Québec yielded a mean SC of 0.482 (95\%CI: $0.475-0.488$ ) for SmaI digests compared with a mean SC of 0.392 (95\% CI: 0.387-0.398) for Kpn I digests $(\mathrm{p}<0.0001$, Wilcoxon rank sum test).

\section{Correlation between clinical and molecular epidemiology data}

Clinical investigation in Estrie identified three cases of campylobacter enteritis that were possibly related to the ingestion of contaminated municipal water, although the source was never definitively established. Kpn I typing confirmed that isolates from these three cases had closely related genotypes and identified two additional such isolates cultured within the same week. In contrast, Sma I typing included only one of these two additional cases within the cluster, and assigned the remaining one to a different cluster that proved to be inconsistent with time and space criteria.

Between July and October, six cases in Estrie were attributed to the ingestion of raw milk. The cases appeared epidemiologically unrelated, as they were all acquired on different farms. Two of these isolates were identified as belonging to a cluster by Sma I digests, but had an SC of 0.80 with $K p n \mathrm{I}$. Two other isolates belonged to a large cluster of 18 isolates identified by Sma I but were classified within two different clusters by $K p n \mathrm{I}$.

\section{Discussion}

PFGE is established as a highly reproducible and discriminatory molecular typing method for a wide range of clinically and epidemiologically significant bacterial pathogens [5]. However, the best strategy for applying PFGE to strain typing of $C$. jejuni has been controversial. The majority of recent reports have relied on Sma I [7, 8, 14, 15] and considered the results obtained with additional enzymes only for selected isolates $[6,11]$. Some laboratories have reported that $K p n$ I digests discriminate among isolates with indistinguishable or closely related Sma I profiles $[6,10,11]$ and the use of more than one restriction endonuclease has been recommended for analysing such isolates $[16,17]$. This study is the first to provide a quantitative analysis of a large set of $C$. jejuni isolates examined with both KpnI and SmaI. The results demonstrate that $K p n$ I digests provide results that are more discriminatory than SmaI and also more consistent with available epidemiological observations.

In assessing discriminatory power, it was observed that epidemiologically unrelated isolates which had indis- 


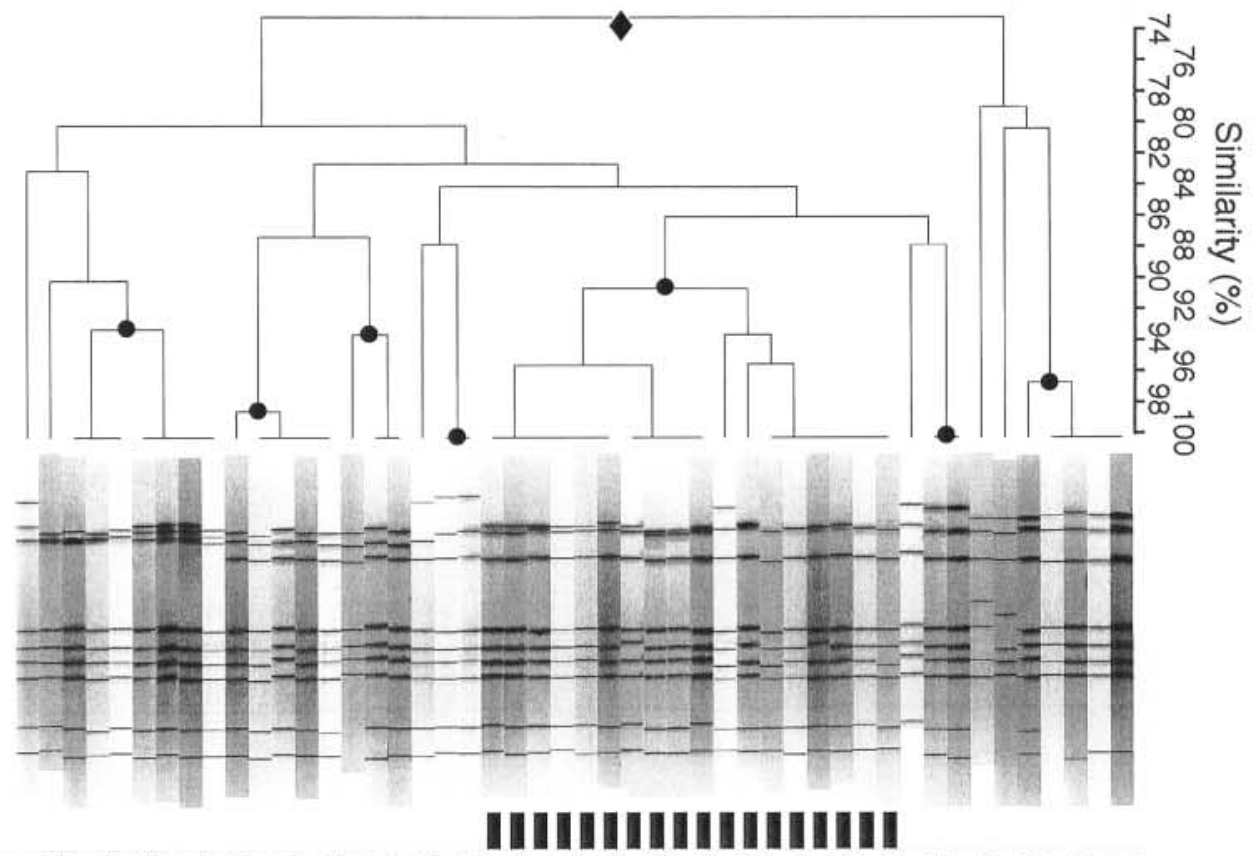

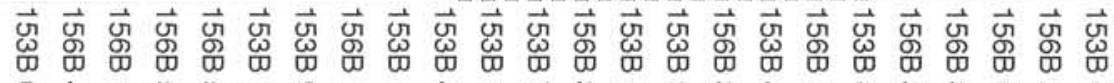

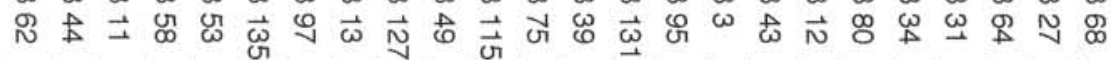

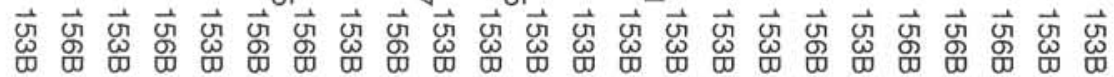

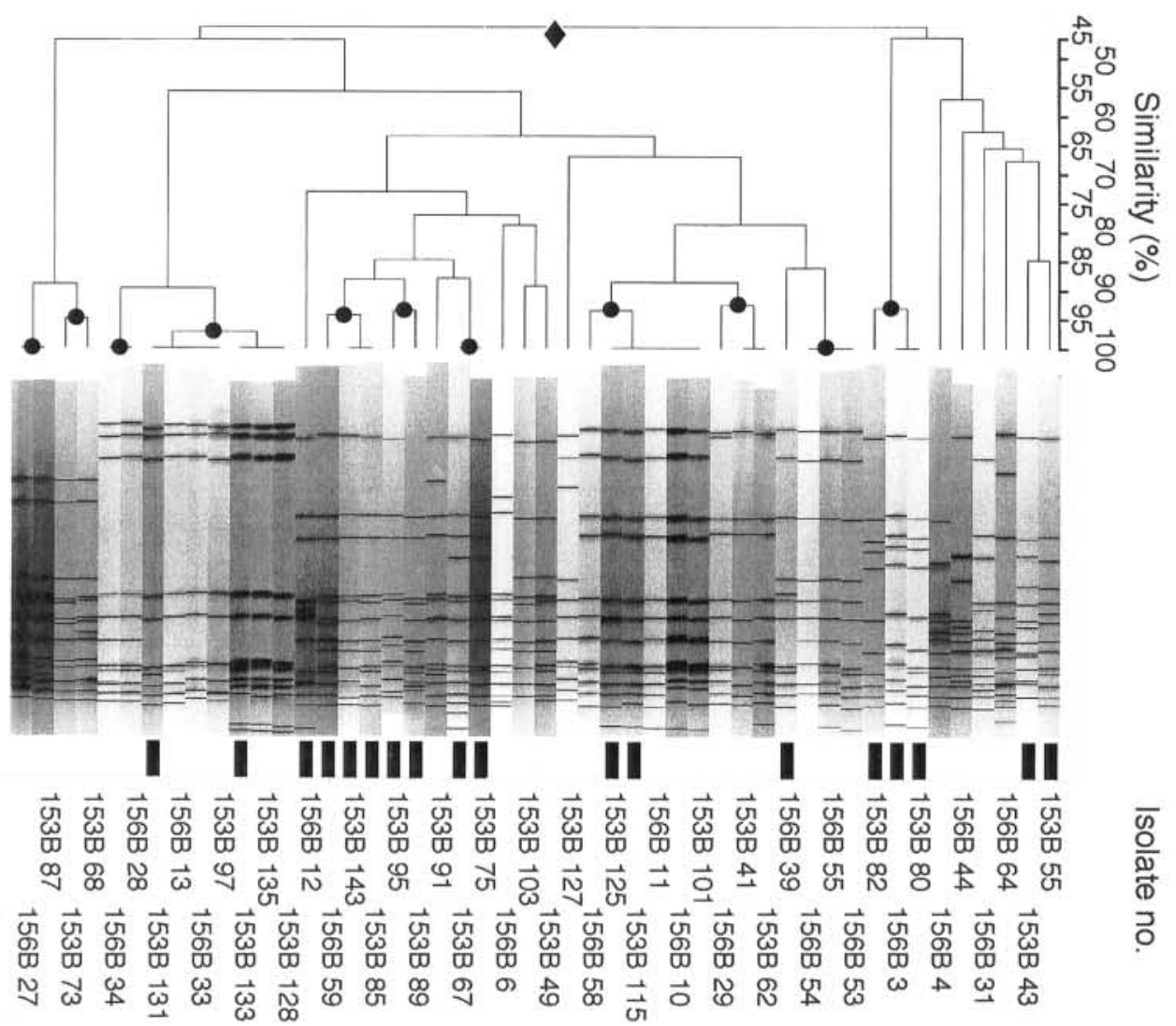

Fig. 1. Dendrograms representing relatedness among PFGE profiles of (a) $S m a \mathrm{I}$ and (b) KpnI digests of 49 isolates of $C$. jejuni. The analysis of the Sma I digests of the 147 study isolates indicated that these 49 isolates were related at the 0.73 level and included a single large cluster of 18 isolates ( $\square$ ) related at the 0.90 level. Analysis of the KpnI patterns of the same 49 isolates demonstrated considerably more diversity, with overall relatedness of 0.43 (b). Moreover, the 18 isolates previously in a single cluster based by the $S m a$ I digests were distributed across the entire dendrogram, with 6 isolates no longer identified as closely related (0.90 level) to any other isolate in that subset. $\bullet$, clusters of isolates related at the 0.90 level; $\bullet$, the root of the dendrogram. 
tinguishable genotypes by Sma I digests could frequently be differentiated by $K p n I$ digests. This may reflect the consistently greater number of restriction fragments generated by $K p n \mathrm{I}$ or, alternatively, that SmaI sites are preferentially found in C. jejuni within loci with restricted genetic variation (e.g., ribosomal operons). As lineages of pathogenic bacteria, including campylobacters, can be distributed worldwide, there is no $a$ priori imperative that all geographically unrelated isolates represent distinct genotypes. However, in practice, for a molecular typing method to be robust and useful it must be able to differentiate $>95 \%$ of epidemiologically unrelated isolates [5]; only KpnI digests met this criterion. Another useful quantitative indication of the discriminatory power of a typing system is the total genetic diversity resolved across a collection of isolates and KpnI resolved appreciably more genetic diversity among the isolates studied.

This study used two approaches to evaluate the validity of the molecular typing results. First, the PFGE analyses were combined with basic epidemiological criteria, i.e., relatedness in time and space. Among 147 isolates, 98 (67\%) were assigned to 27 clusters based on Sma I profiles, and $81(55 \%)$ isolates were assigned to 29 clusters based on KpnI digests. However, 8 $(30 \%)$ of the putative SmaI clusters were inconsistent with the epidemiological criteria compared with 5 (17\%) of the Kpn I clusters. Overall, the total number of clustered isolates decreased to $28 \%$ and $17 \%$, respectively, when epidemiological criteria were combined with the results of strain typing.

This study also specifically examined isolates that had closely related restriction profiles with one endonuclease and re-analysed them with the other endonuclease. More than one-third of isolates that appeared closely related on the basis of SmaI digests were unrelated when analysed by Kpn I digests. Moreover, the 18 isolates assigned to a single large cluster based on Sma I digests were distributed across the entire dendrogram defined by KpnI, with six of the isolates no longer identified as closely related to any other isolate within this subgroup. Finally, clusters that were epidemiologically related to ingestion of raw milk and specific water sources correlated better with the typing results based on KpnI than Sma I. Taken together, these observations strongly support the conclusion that $K p n \mathrm{I}$ is the endonuclease of choice for molecular epidemiological studies of $C$. jejuni.

The combination of KpnI typing and epidemiological (space and time) criteria assigned putative clusters for $49 \%$ of isolates from Estrie and 39\% of isolates from Montréal. This suggests that common source outbreaks of $C$. jejuni may be substantially more frequent than has been suspected on the basis of conventional epidemiological data alone. However, validation of these clusters as instances of recent transmission or common source outbreaks requires additional epidemiological evidence (e.g., case-control study) or molecular typing of a source isolate. The available epidemiological data were insufficient to confirm or refute these putative clusters, as in $41 \%$ of the cases, sources were either missing or explicitly unknown and even those sources proposed were subject to recall bias. Further insights into the epidemiology of sporadic cases of campylobacter enteritis will require prospective application of both clinical and molecular investigations.

This study has several notable limitations. A set of isolates representing an unambiguous outbreak was not available; consequently, the biological reproducibility of C. jejuni molecular typing by Sma I and Kpn I digests could not be formally evaluated. Because of the unreliability of the suspected sources, the study could not demonstrate unambiguously that the greater discriminatory power of KpnI yielded more appropriate epidemiological data. A case-control study combining both clinical and molecular epidemiology data is needed to resolve this issue.

Most of the observed clusters represented two or three isolates. However, this estimate is likely to be artificially low. First, the study analysed only 147 isolates of the 3500 C. jejuni isolates cultured annually in Québec province. Furthermore, many people with enteritis do not seek medical attention and, even among those, only some will have a stool specimen cultured for enteric pathogens. A recent study suggests that among eight cases of campylobacter enteritis in the community only one is included in national surveillance data [4]. Thus, the small clusters observed probably represent only a fraction of the epidemiologically related cases. The time criterion clearly impacted on both the number and the size of the clusters identified. A more stringent requirement, i.e., requiring only 2 or 3 weeks between two sequential genetically related isolates, would have resulted in fewer and smaller clusters, but would not have changed the overall conclusions.

To facilitate studying this relatively large collection of isolates, a computer-based analysis of scanned gel images was applied, rather than visual comparison based on the Tenover criteria [18]. Several issues that proved critical to success with this approach must be emphasised. In each gel, only 13 of 15 available lanes were used, leaving the outermost lanes empty to minimise distortion artifacts. The study included (a) a molecular size standard in every fifth lane, so that when normalising the patterns no isolate was more than two lanes from a standard, and (b) an additional independent standard in every gel so that technical reproducibility could be quantified across the entire series. It is essential that this reproducibility standard is distinct from the molecular size standard; it is helpful to use an isolate of the same species being analysed. The reproducibility patterns were invaluable in validating the user-adjustable parameters present in the ana- 
lytical software; these parameters have a strong influence on the quantitative results and should be noted explicitly.

In conclusion, PFGE analysis of Kpn I digests of $C$. jejuni is more discriminatory and epidemiologically informative than analysis of SmaI digests. Computer analysis of the patterns greatly facilitates the identification of related genotypes among large sets of isolates. As campylobacters are very widely distributed in nature, a combination of approaches will probably be required to identify sources of infection accurately. We propose the use of molecular typing to define clusters of genotypically related isolates, followed quickly by clinical investigation and case-control studies to identify specific common exposures and, finally, selective culturing of food, animal and environmental specimens and molecular typing of the isolates obtained to confirm specific sources. Continuous prospective surveillance studies with this strategy are needed to define the mechanisms of communityacquired $C$. jejuni infection and ultimately to develop new, effective approaches to prevention.

This work was sponsored by the Medical Research Service of the Department of Veterans Affairs (RDA) and by the Centre de Recherche Médicale de l'Université de Sherbrooke (S.M.). We thank Boris Ehrenstein for invaluable assistance with the computer analysis, Janet Senott from the Laboratory Institute of the Massachusetts Department of Public Health for confirming the identification of some C. jejuni isolates, David Carroll for his support and useful suggestions and Alain Lajeunesse for his technical expertise in preparing the figure.

\section{References}

1. Blaser MJ, Taylor DN, Feldman RA. Epidemiology of Campylobacter jejuni infections. Epidemiol Rev 1983; 5: 157-176.

2. Tauxe RV. Epidemiology of Campylobacter jejuni infections in the United States and other industrialized nations. In: Nachamkin I, Blaser MJ, Tompkins LS (eds) Campylobacter jejuni: current status and future trends. Washington, DC, American Society for Microbiology. 1992: 9-19.

3. Blaser MJ. Epidemiologic and clinical features of Campylobacter jejuni infections. J Infect Dis 1997; 176 Suppl 2: S103-S105.

4. Wheeler JG, Sethi D, Cowden JM et al. Study of infectious intestinal disease in England: rates in the community, presenting to general practice, and reported to national surveillance. The Infectious Intestinal Disease Study Executive. BMJ 1999; 318: 1046-1050.

5. Arbeit RD. Laboratory procedures for the epidemiologic analysis of microorganisms. In: Murray PR, Baron EJ, Pfaller MA, Tenover FC, Yolken RH (eds) Manual of clinical microbiology, 7th edn. Washington, DC, ASM Press. 1999: $116-137$.

6. Gibson JR, Fitzgerald C, Owen RJ. Comparison of PFGE, ribotyping and phage-typing in the epidemiological analysis of Campylobacter jejuni serotype HS2 infections. Epidemiol Infect 1995; 115: 215-225.

7. Owen RJ, Sutherland K, Fitzgerald C, Gibson J, Borman P, Stanley J. Molecular subtyping scheme for serotypes HS1 and HS4 of Campylobacter jejuni. J Clin Microbiol 1995; 33: 872-877.

8. Shi ZY, Liu PY, Lau YJ, Lin YH, Hu BS, Tsai HN. Comparison of polymerase chain reaction and pulsed-field gel electrophoresis for the epidemiological typing of Campylobacter jejuni. Diagn Microbiol Infect Dis 1996; 26: 103-108.

9. Swaminathan B, Beebe J, Besser J. Investigation of foodborne and waterborne disease outbreaks. In: Murray PR, Baron EJ, Pfaller MA, Tenover FC, Yolken RH (eds) Manual of clinical microbiology, 7th edn. Washington, DC, ASM Press. 1999: 174-190.

10. Gibson J, Lorenz E, Owen RJ. Lineages within Campylobacter jejuni defined by numerical analysis of pulsed-field gel electrophoretic DNA profiles. J Med Microbiol 1997; 46: 157-163.

11. On SLW, Neilson EM, Engberg J, Madsen M. Validity of Sma I-defined genotypes of Campylobacter jejuni examined by Sal1, KpnI and BamH1 polymorphisms: evidence of identical clones infecting humans, poultry, and cattle. Epidemiol Infect 1998; 120: 231-237.

12. Gibson JR, Sutherland K, Owen RJ. Inhibition of DNAse activity in PFGE analysis of DNA from Campylobacter jejuni. Lett Appl Microbiol 1994; 19: 357-358.

13. Maslow JN, Slutsky AM, Arbeit RD. Application of pulsedfield gel electrophoresis to molecular epidemiology. In: Persing DH, Smith TF, Tenover FC, White TJ (eds) Diagnostic microbiology: principles and applications. Washington, DC, American Society for Microbiology. 1993: 563-572.

14. de Boer P, Duim B, Rigter A, van Der Plas J, Jacobs-Reitsma WF, Wagenaar JA. Computer-assisted analysis and epidemiological value of genotyping methods for Campylobacter jejuni and Campylobacter coli. $J$ Clin Microbiol 2000; 38: 1940-1946.

15. Hudson JA, Nicol C, Wright J, Whyte R, Hasell SK. Seasonal variation of Campylobacter types from human cases, veterinary cases, raw chicken, milk and water. J Appl Microbiol 1999; 87: $115-124$.

16. Wassenaar TM, Newell DG. Genotyping of Campylobacter spp. Appl Environ Microbiol 2000; 66: 1-9.

17. Newell DG, Frost JA, Duim B et al. New developments in the subtyping of Campylobacter species. In: Nachamkin I, Blaser MJ (eds) Campylobacter, 2nd edn. Washington, DC, ASM Press 2000: 27-44.

18 Tenover FC, Arbeit RD, Goering RV et al. Interpreting chromosomal DNA restriction patterns produced by pulsedfield gel electrophoresis: criteria for bacterial strain typing. $J$ Clin Microbiol 1995; 33: 2233-2239. 